\title{
EL QUEHACER CIENTÍFICO VERSUS EL QUEHACER ÁULICO. Buscando rasgos del quehacer científico en libros de texto*
}

\author{
ISLAS, STELLA MARIS y GURIDI, VERÓNICA MARCELA \\ Departamento de Formación Docente. Facultad de Ciencias Exactas. \\ Universidad Nacional del Centro. Pinto 399. 7000 Tandil. Buenos Aires. Argentina. \\ Tel.: (0293) 47100. Fax: (0293) 44431. E-Mail: sislas@exa.unicen.edu.ar.
}

\section{SUMMARY}

Here we outline a search for features of the scientific tasks in the paper-and-pencil and experimental activities proposed in the textbooks mostly used in Tandil. The topics selected were electric current, direct current circuits and electrical current effects. Among other results, it is worth nothing the notable variability found concerning the link between model and reality. There are also some reflections on the lack of emphasis in the conceptual analysis, on the possibility of diversifying the ways for the solution and the discussion on the relevance of mathematical expressions and numerical values.

\section{INTRODUCCIÓN}

Se ha realizado un estudio cualitativo (con un posterior tratamiento cuantitativo), indagando sobre la presencia de rasgos del quehacer científico en las actividades que proponen los libros de texto. Los resultados de tal indagación se usan : a) para comparar las actividades de lápiz y papel con las experimentales en textos para el nivel medio; y b) para comparar las actividades de lápiz y papel en libros para el nivel medio y el nivel superior.

Se consideran dos tipos de actividades:

1) Las de lápiz y papel (ALP), que son las que demandan empleo de leyes cuantitativas o cualitativas, interpretación de gráficos, diagramas y tablas. Usualmente se las denomina problemas y preguntas; pero el término problema, a nuestro entender, alude a una actividad más amplia que la que se toma en los textos. «Un problema es un estado subjetivo de la mente, personal para cada individuo, un desafío, una situación no resuelta, cuya respuesta no es inmediata, que resulta en reflexión y en uso de estrategias conceptuales y procedimentales, provocando un cambio en las estructuras mentales» (Cabral da Costa y Moreira, 1995).

2) Las de experimentación (AE). Usualmente, se las denomina «experiencias» pero hemos preferido llamarlas actividades de experimentación, tomando el sentido en que Beasley concibe las experiencias de enseñanza en laboratorio: «un rango de actividades que abarca desde verdaderas investigaciones experimentales hasta simples ejercicios de verificación y de aprendizaje de destrezas de laboratorio» (Beasley, 1985). 
Anteriores exploraciones con docentes (Islas, 1994) pusieron en evidencia la relevancia que tienen los libros de texto como fuente de la cual los profesores extraen enunciados de problemas y diseños experimentales que luego usan en sus clases; es poco frecuente que sea el propio docente quien elabora esos enunciados y diseños.

Si se aúna el resultado recién citado con los de investigaciones que señalan que en las clases de ciencia se presenta una imagen distorsionada de la misma -por ejemplo, una visión inductivista, con infravaloración de la creatividad, con el descubrimiento científico entendido como ensayo y error- (Hodson, 1985), puede suponerse que los libros de texto contribuyen a tal distorsión.

Nuestra hipótesis es, entonces, que los libros de texto proponen la realización de actividades en las que los rasgos del quehacer científico aparecen con baja frecuencia. Asimismo, suponemos que la presencia de tales rasgos puede detectarse a través de las categorías que más adelante se detallan y que atienden a los rasgos que, según la exploración previa, esperamos encontrar en los textos.

Creemos que esta investigación puede proporcionar información respecto de algunas características de los textos en cuanto ellos cumplen la función recién citada. De manera indirecta, además, los datos obtenidos podrían servir de base para estudios referidos a aspectos más generales de la bibliografía o de la dinámica de las clases.

A nuestro criterio, además, este trabajo puede ser útil como estudio descriptivo de la bibliografía sobre electrodinámica empleada en las físicas básicas, en Tandil, en los años 1995 y 1996.

Para todos los demás universos, sólo debería tomarse con el fin de formular presunciones acerca de los resultados que podrían obtenerse con un estudio similar.

\section{CARACTERÍSTICAS DE LA LABOR CIEN- TIFICA Y SU RELACIÓN CON EL APREN- DIZAJE}

La descripción de las características de la labor científica que se realiza no pretende ser exhaustiva: el propósito de la misma es dejar en claro cuáles son los rasgos del quehacer de los científicos que se relacionan con nuestro objeto de estudio (ALP y AE en libros de texto).

También interesa destacar que entre las actividades propuestas por los textos y las que se concretan en las aulas existe un puente que es el docente. En este trabajo no se indaga, por ejemplo, sobre los criterios que siguen los docentes al momento de seleccionar actividades. El único dato con el que se cuenta es que ellos, como se dijo antes, raramente usan diseños propios para las actividades o bien raramente realizan una selección crítica de los materiales, sino que los extraen de los textos.

En alguna medida, los atributos del quehacer científico deberían imponer su espíritu en las clases de ciencia.
Deberíamos encontrar relación entre las actividades resueltas en los textos y los tópicos que se refieren a continuación.

Los problemas que resuelve el científico son netamente abiertos y envuelven gran cantidad de variables interactuantes. La tarea del investigador comienza realizando un recorte que modeliza la situación. «La construcción de una teoría científica supone simplificaciones tanto en la selección de las variables relevantes como en la formulación de hipótesis acerca de las relaciones entre ellas (Salinas et al., 1995).

La discusión de la compleja relación entre teoría y realidad puede verse como inaccesible para estudiantes de nivel secundario, pero ello no implica la imposibilidad de ofrecerles ocasiones de acercamiento a esa discusión. Uno de los posibles acercamientos comenzaría con el abordaje de situaciones físicas que los estudiantes conocen de su vida cotidiana y ello «haría posible incorporar funcionalmente a los estudiantes en actividades (reflexivas y operativas) de modelado y de emisión de hipótesis» (Salinas et al., 1995).

En la selección de variables relevantes emerge la posibilidad de elección del científico, las tomas de decisión que ponen en juego su libertad y su creatividad. Lejos de ello se encuentra la resolución algorítmica de las ALP y las AE. Al respecto, en su crítica al tinte inductivista de las clases de ciencia, Hodson señala: «Se asume que hay solamente una forma de proceder que no deja lugar a la individualidad y es intolerante con respecto a opiniones atípicas» (Hodson, 1985).

El estudiante debería tener oportunidad de conjugar las informaciones que posee en los distintos dominios para elegir un camino de resolución de una actividad de aprendizaje y no estar siempre limitado a seguir los pasos señalados por un experto.

Los conceptos científicos no se elaboran simplemente como imágenes de los objetos reales y de sus interacciones ni son el resultado de generalizaciones empíricas: «la adquisición de los conceptos de masas puntuales, superficies sin rozamiento, sistema inercial [...], etc. no se produce de forma aristotélica, no surge de mirar los cuerpos e inducir características comunes [...]. La historia alerta a los profesores sobre la necesidad de una aproximación fenomenológica a las idealizaciones: los estudiantes necesitan saber con qué se relacionan las idealizaciones» (Matthews, 1994).

Ya, antes de 1940, Bachelard había señalado la necesidad de que la enseñanza haga explícita la línea de producción espiritual que ha conducido a los resultados del trabajo científico que se pretenden enseñar; de otro modo, al no conocer las razones, el alumno combinará el resultado con las imágenes que le son familiares (Bachelard, 1991).

La construcción de conceptos científicos en el aprendiz resulta de un proceso complejo, que es una evolución «espiralada», un interjuego entre lo concreto y lo abs- 
tracto (en términos vygotskianos) durante el cual el estudiante va interiorizando productos culturales, organizando los conceptos incorporados en un sistema jerárquico (Vygotski, 1991).

La complejidad de este proceso de construcción demanda gran variedad de instancias en las cuales el estudiante se enfrente a interrogantes referidos a cada concepto. En sus intentos por responder a tales interrogantes, se favorecería un progreso dialéctico a través de sucesivos arreglos mejoradores de su estructura cognitiva. En ella, tal como lo han mostrado múltiples investigaciones, están presentes algunos significados erróneos de los términos científicos (ideas alternativas). Acordamos con Moreira que el desarrollo conceptual debería imaginarse «en términos de construcción y discriminación de significados» (Moreira, 1994).

Los esfuerzos de los científicos en sus intentos por explicar los fenómenos físicos dan lugar a formulaciones matemáticas que se relacionan entre sí de acuerdo con las leyes de la lógica. Pero, por sobre todo, estas expresiones formales son descripciones de acontecimientos naturales y, como tales, han de manejarlas tanto el científico como el profesor de física y sus alumnos. En la caracterización de los estudios de epistemología e historia de la física para futuros docentes que realizan Salinas y Cudmani (1993), para clarificar la relación entre la matemática y la física, se destaca que «el rigor cuantitativo requiere imprescindiblemente del rigor cualitativo, conceptual, para llenar de significado una forma que, de otro modo, está vacía de contenido fáctico».

En las Feynman Lectures on Physics, encontramos: «Si tenemos el medio de conocer qué está realmente sucediendo en una circunstancia dada sin resolver efectivamente las ecuaciones, habremos comprendido las ecuaciones.» (Feynman, 1987). Esto es: si se quiere llegar a un acertado manejo de los formalismos, deben correlacionarse éstos con sus referentes fácticos.

La escuela media es el ámbito en el cual el estudiante debe construir aquellos conceptos de la física que le permitan interpretar adecuadamente la descripción matemática de los mismos.

\section{METODOLOGÍA}

Se pueden distinguir dos instancias: una, de índole cualitativa, con formulación de categorías; y otra, cuantitativa, en la que se confeccionaron tablas de frecuencias para luego efectuar dos comparaciones:

- Las actividades de lápiz y papel (ALP) en textos de nivel medio (NM) comparadas con las que aparecen en textos de nivel superior (NS).

- Las actividades de lápiz y papel (ALP) comparadas con las actividades experimentales (AE) en textos de nivel medio (NM).

\section{Selección de libros de texto}

En un trabajo anterior (Islas, 1994), se solicitó a profesores de escuelas medias de Tandil y de físicas básicas en la Universidad Nacional del Centro que informaran sobre los textos que ellos empleaban como guía para organizar sus clases y que recomendaban a sus alumnos para preparar los temas. De la lista confeccionada en tal oportunidad se extrajeron aquéllos que tratan la electrodinámica y, dentro de este grupo, se tomaron los textos cuyos autores brindan pautas para realizar las actividades (ya que en este trabajo no se analizan sólo enunciados, sino también las sugerencias que da el autor para trabajar a partir de ellos).

Se realizó una exploración previa sobre un grupo de libros de texto (elegidos sobre la base de datos proporcionados por docentes) que permitió concretar la selección de textos y de temas, y elaborar las categorías de estudio que se detallan más adelante.

El estudio que aquí presentamos entra en lo que Samaja (1994) denomina de diseño descriptivo y, dentro de ellos, es de los que emplea muestras no aleatorias. Está claro que este tipo de muestreo no permite calcular la probabilidad de la representatividad de la muestra, esto es, no permite conocer el riesgo que se corre al afirmar que el comportamiento del universo será análogo al de la muestra.

Ahora bien, acordamos con Samaja que «el hecho de que no se pueda asignar un valor numérico a la incertidumbre no quiere decir que la incertidumbre sea grande» (Samaja, 1994, p. 273).

Puede ponderarse tal incertidumbre empleando, para la selección muestral, criterios sustantivos (y no formales), que están fundados en lo que se sabe acerca del tema que se está estudiando (en este caso, los textos de física empleados en Tandil para electrodinámica en los niveles medio y superior) y lo que se sabe acerca de cómo se obtuvo la muestra.

De acuerdo con los criterios que se han detallado en párrafos anteriores, la muestra seleccionada consta de cinco textos, a saber:

Texto 1: Maiztegui-Boido. Física 4. Ed. Kapelusz. Buenos Aires. 1993.

Texto 2: Resnick- Halliday. Física 2. Ed. CECSA. México. 1979.

Texto 3: Stollberg-Hill. Física: Fundamentos y fronteras. Ed. Publicaciones Cultural, SA. México. 1984.

Texto 4: Tipler. Física 2. Ed. Reverté. Madrid. 1979.

Texto 5: Tricárico-Bazo. AZ editora. Buenos Aires. 1994. 


\section{Selección temática}

Se buscó un grupo de temas cuyo tratamiento fuera frecuente en las escuelas medias: es sabido que los recortes que los docentes efectúan en sus programas de contenidos son de notable variabilidad; sin embargo, anteriores exploraciones han mostrado que estos temas están presentes en la mayoría de las clases sobre electromagnetismo.

Se tomó electrodinámica y sólo el «primer escalón» del electromagnetismo (efecto Oersted); se conformó así un grupo de temas para los cuales es común que se propongan ambos tipos de actividades (ALP y AE).

Los temas trabajados fueron: corriente eléctrica, circuitos de corriente continua y efectos de la corriente eléctrica. La secuencia de contenidos es relativamente uniforme en los textos para escuela media; no así en los del nivel superior. En éstos se trabajó desde la ley de Ohm hasta las leyes de Ampère y Biot-Savart (incluyendo, claro está, los circuitos óhmicos y leyes de Kirchoff). Se estudió lo relativo al efecto Oersted pero no a la acción de un campo magnético sobre una corriente.

Así, resultaron analizadas 49 actividades de lápiz y papel (ALP) y 26 actividades experimentales (AE). Cabe aclarar que las AE corresponden a textos de nivel medio, dado que en los textos de nivel superior que se tomaron para este trabajo no se propone este tipo de actividades.

\section{Tratamiento de datos}

Elaboramos una lista de categorías que describen las ALP y las AE sobre la base de las consideraciones teóricas apuntadas en el recorte del problema, siguiendo los lineamientos sugeridos por la metodología cualitativa.

Durante el análisis de las categorías, se vio la necesidad de reformular algunas de ellas y de eliminar otras por su escasa representatividad en los textos. Se procedió de acuerdo con la adaptación del muestreo teórico (de Glasser- Strauss), que aparece fundamentada en el capítulo «El trabajo con los datos» de la obra de Taylor y Bogdan (1992, p. 152 y sig.).

Se han tomado algunas prácticas similares a las del «análisis de contenido» de Ander Egg (1990), pero no presentamos nuestros resultados como emergentes del uso de esa técnica, dadas las sucesivas adaptaciones que hicimos de la misma.

La elección de categorías requirió de un diseño específico para este trabajo, ya que, si bien hay estudios referidos al empleo de libros de texto, éstos atienden a poblaciones o a temáticas diferentes a las de este trabajo o bien se valen de recursos metodológicos diferentes. Por ejemplo, hay estudios realizados en el estado de Michoacán (México) sobre el uso de textos de ciencias naturales (Gutiérrez Vázquez et al., 1993); sobre mecá- nica elemental en textos usados en la ciudad de Tandil (Islas, 1994); sobre leyes de Newton en libros de texto en la ciudad de Río Cuarto (Vicario et al., 1995). En otros, la diferencia es de corte metodológico y, así, tenemos como ejemplos: análisis de entrevistas (Otero, 1990), estudio de estructuras en textos (BrinconesOtero, 1994), análisis de explicaciones en los textos (Aguirre, 1983), uso del análisis de contenido para el concepto de energía en libros de texto (Michinel y D’Alessandro, 1994).

Si bien se han tomado algunas ideas sugeridas por otras investigaciones, la relevancia que adquieren las mismas en nuestro trabajo no es suficiente para calificarlas como fuentes.

La lista de categorías diseñada para este trabajo fue contrastada con la colaboración de tres investigadores del equipo del cual formamos parte: se cotejaron los resultados señalados por ellos con los nuestros, encontrando porcentajes de concordancia que afirmaron la decisión de tomar algunas categorías, y disidencias de interpretación que orientaron la reformulación de otras. Ha de tenerse presente que esto no valida la extensión de las conclusiones de este trabajo a todos los libros de texto; sólo permite fundamentar la descripción de las obras analizadas, con la circunscripción temática ya señalada.

Por tratarse de un instrumento no estandarizado, consideramos oportuno presentar detalles acerca de los criterios con que se han valorado las categorías en los textos.

\section{C1: El recorte que modeliza la situación problemática} está explicitado.
Valores:
$\left.\mathrm{a}_{1}\right)$ Sí
$\left.b_{1}\right)$ No
$\left.\mathrm{c}_{1}\right)$ Algo

La explicitación de hipótesis simplificatorias y de límites de validez de leyes y campos fácticos de aplicación se buscaron no sólo en la actividad sino también en el contexto del desarrollo temático. Ejemplo: en las actividades sobre la ley de Ohm se eligió el valor «sí» aun en los casos en que el marco de validez de la ley no estaba indicado en el desarrollo (se tomó «sí», pues tal marco estaba señalado en otra parte del texto).

\section{C2: El recorte simplificatorio deviene en omisión de} variables relevantes de la situación real.

Valores: $a_{2}$ ) Sí $\quad b_{2}$ ) No $c_{2}$ ) Algo $d_{2}$ ) No corresponde

Se consideraron como omisiones de variables relevantes, por ejemplo: no tener en cuenta la influencia de la temperatura en la resistencia de un conductor (en aquellos casos en los que la variación en la resistencia debida al cambio en la temperatura del conductor es considerable respecto del error propio de las mediciones); o bien tomar con validez estricta la igualdad entre trabajo y energía cuando existe disipación notable de la segunda en forma de calor. 
C3: Se da mayor relevancia al cálculo que al análisis físico de la situación.

Valores: $a_{3}$ ) Sí $\quad b_{3}$ ) No $\quad c_{3}$ ) Algo $\quad d_{3}$ ) No corresponde

En las ALP se señaló que esto ocurre cuando lo único que se hace es utilizar fórmulas. En casos en los que se agrega algún breve comentario (por ejemplo, justificación de la elección de las fórmulas), se consideró el valor «algo». En las AE se adjudicó el valor «sí» cuando la formalización matemática de una ley no está precedida por algún análisis de las variables que intervienen en el fenómeno abordado. El valor «no corresponde» aparece cuando la actividad es de índole cualitativa.

C4: Las estrategias de resolución se muestran explícitamente señaladas.
Valores:
$\left.\mathrm{a}_{4}\right)$ Sí
$\left.\mathrm{b}_{4}\right) \mathrm{No}$
$\mathrm{c}_{4}$ ) Algo

Por «explícitamente señaladas» entendemos aquellas demarcaciones tales que el alumno no puede seguir otro camino distinto al que recorre el autor. En el caso de las ALP, el valor «algo» se computa si se presentan solamente las ecuaciones a utilizar, pero no se realizan transformaciones matemáticas ni cálculos posteriores.

C5: Se analiza la aceptabilidad de valores numéricos en el marco particular de la situación.

Valores: $a_{5}$ ) Sí $\quad b_{5}$ ) No $\quad c_{5}$ ) Algo $d_{5}$ ) No corresponde

Consideramos que, con respecto a esta categoría, sólo es pertinente la siguiente aclaración: principalmente en los textos de nivel superior, aparecen ALP en las que no se demanda la realización de cálculos (no hay valores de los datos) sino que se solicita encontrar la expresión que permitiría encontrar un valor. Se tuvo en cuenta si esta expresión se presenta directamente $\left(\right.$ valor $\mathrm{b}_{5}$ ) o si se hace alguna discusión acerca de la aplicabilidad de la misma (valor $c_{5}$ ). Se tomó el valor «no corresponde» $\left(d_{5}\right)$ en el caso de AE de tipo cualitativo y en aquellas ALP en las cuales la ausencia de datos previos o saberes cotidianos imposibilita el análisis de aceptabilidad.

\section{C6: Empleo de la teoría relacionada con la actividad.}

Valores: $a_{6}$ ): Se emplea para formular predicciones. $\mathrm{b}_{6}$ ): Se emplea para explicar resultados.

$\mathrm{c}_{6}$ ): Se emplea para organizar la tarea.

$\left.\mathrm{d}_{6}\right)$ : No se manifiesta.

El valor «no se manifiesta» corresponde a aquellos casos en los que no se encuentra un puente entre la teoría y la práctica.

Cabe aclarar que, en esta categoría, los valores no son mutuamente excluyentes.

C7: Se analiza el significado de los conceptos a los que se hace referencia.
Valores:
$\left.\mathrm{a}_{7}\right)$ Sí
$\left.\mathrm{b}_{7}\right) \mathrm{No}$
$\left.\mathrm{c}_{7}\right)$ Algo

C8: El lenguaje científico se utiliza, además de en su forma verbal, en sus expresiones.

Valores: $\mathrm{a}_{8}$ ) Gráfica

$b_{8}$ ) Algebraica

$\mathrm{c}_{8}$ ) Por tablas

$\mathrm{d}_{8}$ ) Por diagramas o esquemas

Aquí tampoco los valores son excluyentes.

C9: Hay demanda de contrastación de hipótesis.

Valores: $\quad a_{9}$ ) Sí $\quad b_{9}$ ) No $\quad c_{9}$ ) No corresponde

Se consigna el valor «no corresponde» cuando, por estar el desarrollo temático muy acotado, no da lugar a que el alumno elabore conjeturas a partir del enunciado de la cuestión. En los textos de nivel superior, el desarrollo temático es, en general, lo suficientemente amplio como para que a través de las cuestiones pueda pedirse al estudiante que formule y contraste una hipótesis.

C10: Se alude explícitamente al modo en que trabajan los científicos.

Valores: $\quad a_{10}$ ) Sí $\quad b_{10}$ ) No $\quad c_{10}$ ) Algo

El valor «sí» corresponde a propuestas en las que se hace, por ejemplo, una comparación entre el desarrollo de la actividad y el estilo con que la resuelve un científico (ya sea en cuanto a las preguntas que se formulan, como a las consideraciones de cálculo de incertezas, a la determinación de variables relevantes, a la pertinencia de revisiones, etc.) o a propuestas en las que se realizan referencias históricas. Se tomó el valor «sí» cuando la alusión se incluye como comentario dentro de la actividad.

\section{Resultados}

La comparación se realizó por categoría, cotejando los porcentuales obtenidos para cada valor de las mismas. Luego se observó el total de valores registrados en busca de tendencias que facilitaran la comparación.

Los resultados se presentan en las tablas I y II. En ellas, se escriben en el comienzo de cada línea las palabras clave para cada categoría, con el fin de facilitar la lectura.

Luego de cada tabla, se ofrecen breves comentarios sobre lo que se muestra para cada categoría.

Comparación entre los textos de nivel medio (NM) y superior (NS) para actividades de lápiz y papel (ALP)

La tabla I muestra, para cada valor de las distintas categorías, los porcentajes obtenidos en nivel medio y nivel superior.

Se han sombreado las líneas en las cuales son máximas las frecuencias de un mismo valor. Esto corresponde a 


\begin{tabular}{|c|c|c|c|}
\hline CATEGORÍA & NIVEL & $\begin{array}{c}\text { Medio } \\
\text { (NM) }\end{array}$ & $\begin{array}{c}\text { Superior } \\
\text { (NS) }\end{array}$ \\
\hline \multirow{3}{*}{$\begin{array}{l}\text { Categoría } 1 \\
\text { (Recorte explicitado) }\end{array}$} & $\mathrm{a}_{1}$ : Sí & 50 & 87 \\
\hline & $\mathrm{b}_{1}:$ No & 28 & 6 \\
\hline & $\mathrm{c}_{1}:$ Algo & 23 & 6 \\
\hline \multirow{4}{*}{$\begin{array}{l}\text { Categoría } 2 \\
\text { (Omisión de variables) }\end{array}$} & $\mathrm{a}_{2}:$ Sí & 11 & 0 \\
\hline & $\mathrm{b}_{2}: \mathrm{No}$ & 67 & 97 \\
\hline & $\mathrm{c}_{2}:$ Algo & 11 & 0 \\
\hline & $\mathrm{d}_{2}:$ No corresponde & 11 & 3 \\
\hline \multirow{4}{*}{$\begin{array}{l}\text { Categoría } 3 \\
\text { (Relevancia dada al cálculo) }\end{array}$} & $\mathrm{a}_{3}:$ Sí & 78 & 39 \\
\hline & $\mathrm{b}_{3}$ : No & 11 & 32 \\
\hline & $\mathrm{c}_{3}:$ Algo & 11 & 29 \\
\hline & $\mathrm{d}_{3}:$ No corresponde & 0 & 0 \\
\hline \multirow{3}{*}{$\begin{array}{l}\text { Categoría } 4 \\
\text { (Estrategias explicitadas) }\end{array}$} & $\mathrm{a}_{4}: \mathrm{Sí}^{\prime}$ & 94 & 84 \\
\hline & $\mathrm{b}_{4}: \mathrm{No}$ & 0 & 0 \\
\hline & $\mathrm{c}_{4}:$ Algo & 6 & 16 \\
\hline \multirow{4}{*}{$\begin{array}{l}\text { Categoría } 5 \\
\text { (Aceptabilidad de valores) }\end{array}$} & $\mathrm{a}_{5}: \mathrm{Sí}^{\prime}$ & 6 & 32 \\
\hline & $\mathrm{b}_{5}: \mathrm{No}$ & 55 & 26 \\
\hline & $\mathrm{c}_{5}:$ Algo & 0 & 35 \\
\hline & $\mathrm{d}_{5}:$ No corresponde & 39 & 6 \\
\hline \multirow{4}{*}{$\begin{array}{l}\text { Categoría } 6 \\
\text { (Uso de teoría) }\end{array}$} & $\mathrm{a}_{6}:$ Formular predicciones & 0 & 26 \\
\hline & $\mathrm{b}_{6}:$ Explicar resultados & 17 & 84 \\
\hline & $\mathrm{c}_{6}$ : Organizar la tarea & 78 & 68 \\
\hline & $\mathrm{d}_{6}:$ No se manifiesta & 11 & 0 \\
\hline \multirow{3}{*}{$\begin{array}{l}\text { Categoría } 7 \\
\text { (Análisis conceptual) }\end{array}$} & $\mathrm{a}_{7}$ : Sí & 11 & 23 \\
\hline & $\mathrm{b}_{7}: \mathrm{No}$ & 72 & 39 \\
\hline & $\mathrm{c}_{7}:$ Algo & 17 & 39 \\
\hline \multirow{4}{*}{$\begin{array}{l}\text { Categoría } 8 \\
\text { (Uso del lenguaje) }\end{array}$} & $\mathrm{a}_{8}$ : Gráfico & 0 & 13 \\
\hline & $\mathrm{b}_{8}$ : Algebraico & 94 & 100 \\
\hline & $\mathrm{c}_{8}:$ Tablas & 0 & 0 \\
\hline & $\mathrm{d}_{8}$ : Diagramas o esquemas & 33 & 65 \\
\hline \multirow{3}{*}{$\begin{array}{l}\text { Categoría } 9 \\
\text { (Contrastación de hipótesis) }\end{array}$} & $\mathrm{a}_{9}:$ Sí & 0 & 26 \\
\hline & $b_{9}:$ No & 6 & 74 \\
\hline & $\mathrm{c}_{9}:$ No corresponde & 94 & 0 \\
\hline \multirow{3}{*}{$\begin{array}{l}\text { Categoría } 10 \\
\text { (Alusión al trabajo científico) }\end{array}$} & $\mathrm{a}_{10}: \mathrm{Sí}$ & 0 & 3 \\
\hline & $\mathrm{b}_{10}: \mathrm{No}$ & 100 & 97 \\
\hline & $\mathrm{c}_{10}:$ Algo & 0 & 0 \\
\hline
\end{tabular}

casos en los que se da el mismo valor («sí», «no», etc.) en el nivel medio y en el nivel superior.

Se exceptúa el sombreado en la categoría 5 (debido a que en ésta aparece una notable variabilidad en los valores registrados) y en la número 9 (para la cual se presenta una situación diferente, según el nivel del texto). En las restantes, es interesante notar que la máxima frecuencia se da, en cada categoría, para un mismo valor en el NM y en el NS.

Una lectura más minuciosa de la tabla I permite observar, además, que: exceptuando las categorías 5 y 9 (las mismas en que se excluyó el sombreado de coincidencia 
de frecuencias máximas), si se toman los porcentajes en cada categoría y se los ordena de mayor a menor, se tendrá el mismo ordenamiento de líneas para ambos niveles (NM y NS).

C1: En ambos niveles, los porcentajes más elevados se dan para el valor $\mathrm{a}_{1}$ : el recorte está explicitado. El rigor aparece con mayor frecuencia en textos de nivel superior.

$C 2$ : Hay una notable acumulación de casos en los que no hay omisión de variables relevantes de la situación real. También aquí se registra la misma tendencia: el porcentaje es más elevado en textos de NS. En los textos de nivel medio, la mayoría de las omisiones se refieren al ya citado ejemplo de que no se considera que la resistencia depende de la temperatura.

C3: En la mayoría de los casos (78\% en NM; 39\% en NS) se da mayor relevancia al cálculo que al análisis físico de la situación. Con respecto al valor $b_{3}$ (no se da mayor relevancia al cálculo que al análisis físico), sólo ocurre en un $11 \%$ en el NM y en un $32 \%$ en el NS.

C4: Se observa una gran similitud entre los porcentajes obtenidos para ambos niveles (94\% y $84 \%$ para NM y NS, respectivamente) en estrategias normalizadas. Son muy pocos los problemas para los cuales el alumno puede elegir caminos alternativos de resolución.

C5: Hay una heterogeneidad en los porcentuales mayor que la esperada. En textos para NM es menos frecuente realizar análisis de aceptabilidad de resultados que en textos para NS.

C6: El uso de la teoría aparece generalmente para organizar la tarea y (en textos para NS) aparece para explicar resultados, en tanto que es menos frecuente que la teoría se emplee para formular predicciones, especialmente para NM.

C7: En ambos niveles, el análisis conceptual aparece con baja frecuencia.

C8: El lenguaje algebraico es utilizado en la casi totalidad de las ALP analizadas. Sólo en una de tales actividades no se utiliza este tipo de lenguaje. En ciertos casos este lenguaje se combina con la realización de diagramas o esquemas. En sólo cuatro actividades del NS se propone la realización de gráficos cartesianos. En ningún caso se emplean tablas.

C9: Se pide comprobación de hipótesis en un $26 \%$ de ALP para NS, pero en el $74 \%$ restante, si bien están dadas las condiciones de desarrollo temático para formularlas, no se las requiere. En los textos de NM, en cambio, por ser el desarrollo temático bastante sucinto, no se evidencian posibilidades de que el estudiante formule y contraste hipótesis.

C10: Se consignó el valor «sí» para un solo caso de ALP en el NS (3\% sobre el total), dado que se realizó una referencia histórica muy breve.
Comparación entre actividades de lápiz y papel (ALP) y actividades experimentales $(A E)$ para textos de nivel medio (NM)

Los resultados obtenidos se muestran en la tabla II

Puede notarse que el sombreado de líneas de coincidencia de frecuencias máximas aparece solamente en cuatro categorías: en las restantes, los máximos para cada categoría se dan con diferentes valores, según se trate de actividades experimentales o de lápiz y papel. Reviste carácter diferente la coincidencia en la categoría 9: si bien se da la frecuencia máxima en el valor «no corresponde» en ambas columnas, puede verse que esa frecuencia máxima se repite en la segunda columna, para otro valor de la variable.

No se verifica, en esta tabla, que el ordenamiento de frecuencias sea el mismo para la mayoría de las categorías.

También, en este caso, se efectuó una comparación por categoría, registrándose lo siguiente:

C1: Con respecto a las $\mathrm{AE}$, es más raro encontrar explicitado el recorte si se comparan con las ALP.

C2: En las AE se realizan más omisiones de variables relevantes que en las ALP. Cuando se realiza el diseño experimental, se practica un recorte que pocas veces se explicita. Son muy pocos los casos en los cuales se toman en cuenta los errores de medición; y es preciso contemplar que el análisis de error brinda parámetros que orientan al científico para clasificar variables en relevantes y no relevantes.

C3: La única observación que puede hacerse respecto a la relevancia otorgada al cálculo es que hay una evidente variabilidad en todos los valores.

C4: En la mayoría de las actividades ( $85 \%$ para AE y $94 \%$ para ALP), las estrategias aparecen claramente señaladas. Se deja poco espacio para la iniciativa del alumno.

C5: En pocos casos de AE se analiza la aceptabilidad de valores numéricos, pero aquí debe tenerse en cuenta que hay muchas experiencias que son de índole cualitativa. En consecuencia, no se espera que se realice este tipo de análisis.

C6: El empleo de teoría se circunscribe a la explicación de resultados para las AE, y para organizar la tarea en el caso de las ALP. Se registran porcentajes muy bajos para la formulación de predicciones.

C7: El análisis conceptual es poco frecuente para ambos tipos de actividades.

C8: Para las ALP predomina el uso del lenguaje algebraico y para las AE, el de diagramas o esquemas.

C9: La demanda de contrastación de hipótesis aparece sólo en el $15 \%$ de los casos analizados de AE. Ya se 


\begin{tabular}{|c|c|c|c|}
\hline CATEGORÍA & NIVEL & ALP & $\mathbf{A E}$ \\
\hline \multirow{3}{*}{$\begin{array}{l}\text { Categoría } 1 \\
\text { (Recorte explicitado) }\end{array}$} & $\mathrm{a}_{1}$ : Sí & 50 & 6 \\
\hline & $b_{1}:$ No & 28 & 92 \\
\hline & $\mathrm{c}_{1}$ : Algo & 20 & 2 \\
\hline \multirow{4}{*}{$\begin{array}{l}\text { Categoría } 2 \\
\text { (Omisión de variables) }\end{array}$} & $\mathrm{a}_{2}:$ Sí & 11 & 50 \\
\hline & $\mathrm{b}_{2}:$ No & 67 & 46 \\
\hline & $\mathrm{c}_{2}:$ Algo & 11 & 0 \\
\hline & $\mathrm{d}_{2}:$ No corresponde & 11 & 4 \\
\hline \multirow{4}{*}{$\begin{array}{l}\text { Categoría } 3 \\
\text { (Relevancia dada al cálculo) }\end{array}$} & $\mathrm{a}_{3}:$ Sí & 78 & 0 \\
\hline & $\mathrm{b}_{3}:$ No & 11 & 35 \\
\hline & $\mathrm{c}_{3}:$ Algo & 11 & 12 \\
\hline & $\mathrm{d}_{3}:$ No corresponde & 0 & 54 \\
\hline \multirow{3}{*}{$\begin{array}{l}\text { Categoría } 4 \\
\text { (Estrategias explicitadas) }\end{array}$} & $\mathrm{a}_{4}:$ Sí & 94 & 85 \\
\hline & $\mathrm{b}_{4}: \mathrm{No}$ & 0 & 0 \\
\hline & $\mathrm{c}_{4}:$ Algo & 6 & 14 \\
\hline \multirow{4}{*}{$\begin{array}{l}\text { Categoría } 5 \\
\text { (Aceptabilidad de valores) }\end{array}$} & $a_{5}:$ Sí & 6 & 0 \\
\hline & $\mathrm{b}_{5}:$ No & 55 & 19 \\
\hline & $\mathrm{c}_{5}:$ Algo & 0 & 0 \\
\hline & $\mathrm{d}_{5}:$ No corresponde & 39 & 80 \\
\hline \multirow{4}{*}{$\begin{array}{l}\text { Categoría } 6 \\
\text { (Uso de la teoría) }\end{array}$} & $\mathrm{a}_{6}:$ Formular predicciones & 0 & 12 \\
\hline & $\mathrm{b}_{6}:$ Explicar resultados & 17 & 69 \\
\hline & $\mathrm{c}_{6}$ : Organizar la tarea & 78 & 31 \\
\hline & $\mathrm{d}_{6}$ : No se manifiesta & 11 & 15 \\
\hline \multirow{3}{*}{$\begin{array}{l}\text { Categoría } 7 \\
\text { (Análisis conceptual) }\end{array}$} & $\mathrm{a}_{7}$ : Sí & 11 & 31 \\
\hline & $b_{7}:$ No & 72 & 58 \\
\hline & $\mathrm{c}_{7}:$ Algo & 17 & 12 \\
\hline \multirow{4}{*}{$\begin{array}{l}\text { Categoría } 8 \\
\text { (Uso del lenguaje) }\end{array}$} & $\mathrm{a}_{8}$ : Gráfico & 0 & 12 \\
\hline & $b_{8}:$ Algebraico & 94 & 15 \\
\hline & $\mathrm{c}_{8}$ : Tablas & 0 & 19 \\
\hline & $\mathrm{d}_{8}$ : Diagramas o esquemas & 33 & 85 \\
\hline \multirow{3}{*}{$\begin{array}{l}\text { Categoría } 9 \\
\text { (Contrastación de hipótesis) }\end{array}$} & $\mathrm{a}_{9}:$ Sí & 0 & 15 \\
\hline & $b_{9}$ : No & 6 & 42 \\
\hline & $c_{9}:$ No corresponde & 94 & 42 \\
\hline \multirow{3}{*}{$\begin{array}{l}\text { Categoría } 10 \\
\text { (Alusión al trabajo científico) }\end{array}$} & $\mathrm{a}_{10}:$ Sí & 0 & 3 \\
\hline & $\mathrm{b}_{10}:$ No & 100 & 97 \\
\hline & $\mathrm{c}_{10}:$ Algo & 0 & 0 \\
\hline
\end{tabular}

señaló que esta demanda no se registra en las ALP para NM.

C10: En cuanto a la alusión al trabajo científico, sólo se ha encontrado un caso de AE en el que se efectúa una referencia histórica: se describe cómo llevó a cabo el experimento el científico que lo realizó por primera vez. Esto constituye un 3\% sobre el total (porcentaje muy bajo). Para las ALP no se han registrado casos en los que se realiza alusión. 


\section{CONCLUSIONES}

Los títulos relativos a la modelización de situaciones muestran una variabilidad que sugiere que, principalmente en el nivel medio, no prima el interés en que el estudiante conozca cómo se lleva a cabo el modelado y cómo se establece el vínculo entre teoría y realidad. «Creemos estar haciendo una presentación elemental simplemente porque eludimos la complejidad matemática en la parte formal, pero fallamos en transmitir algo esencial: nuestro modo de razonamiento, nuestro universo mental. Dejamos tácitas las pautas generales por las cuales un físico, para determinado problema, puede, por ejemplo, hacer un modelo en el cual los caballos son esféricos y, lo que es peor, sacar una conclusión que coincida con lo observado.» (Grünfeld, 1991).

Lo anterior permite preguntarse acerca de la conexión entre teoría y realidad que se promueve en los estudiantes. Esta pregunta tiene su base, además, en las siguientes observaciones:

- Se registra mayoritariamente un empleo de teoría en una forma que podría calificarse de «mínimo indispensable» (organizar la tarea o justificar resultados). Creemos que se podría dar con mayor frecuencia la demanda de contraste de hipótesis y el uso de la teoría para formular predicciones, caracteres del trabajo científico que ya fueron destacados cuando se realizó el recorte del problema.

- Son muy pocos los casos en los que se analizan los conceptos que son utilizados. Entendemos que las actividades (tanto las ALP como las AE) deberían tener, entre otros objetivos, el de favorecer la internalización de conceptos. ¿Cuáles serían, si no, las situaciones en las que el estudiante puede poner en juego sus saberes para trabajar con conceptos que son nuevos para él?

Ya se mencionó con anterioridad la conveniencia de que el estudiante aborde situaciones que le son familiares.

\section{REFERENCIAS BIBLIOGRÁFICAS}

ANDER EGG, E. (1990). Técnicas de investigación social. Buenos Aires: Humanitas.

AGUIRRE DE CÁRCER, I. (1983). Dificultades en la comprensión de las explicaciones de los libros de texto de física. Enseñanza de las Ciencias, 1(2), pp. 92-98.

BACHELARD, G. (1991). La formación del espíritu científico. México: Siglo XXI Editores.

BEASLEY, W. (1985). Improving Student Laboratory Performance: How Much Practice Makes Perfect? Science Education, 69(4), pp. 567-576.
En otro estudio, cuyos resultados no se consignan aquí, se estudió la relación de las ALP y las AE con saberes de la vida cotidiana, y se encontró que el $73 \%$ de las ALP y el $77 \%$ de las AE no presentan un vínculo con saberes cotidianos.

El análisis de aceptabilidad de valores numéricos, contemplando las incertezas en las mediciones -en aquellos casos que es pertinente- debería aparecer (en nuestra opinión) con mayor frecuencia que la observada. Este tipo de análisis es una de las instancias de control de ajuste del modelo a los fenómenos que representa.

Una observación curiosa puede hacerse respecto de las ALP: si bien no se explica cómo se hace el paso de la situación real a su presentación formal, esta última tiene preponderancia sobre el análisis físico de la situación.

Por otra parte, las estrategias de resolución aparecen muy pautadas y esta tendencia es uniforme para ambos tipos de actividades, lo que permite concluir que, por un lado, no se da espacio a la iniciativa y a la creatividad del alumno y, por otro, se contribuye a distorsionar la imagen del científico, presentando su trabajo como algo muy estructurado y algorítmico (en casos extremos, rutinario). Esto se refuerza con el hecho de que, en sólo dos de setenta y cinco actividades analizadas, está presente la alusión al modo en que un científico las lleva a cabo.

Esta falta de diversidad en caminos de resolución se manifiesta también en la elección del lenguaje a usar: son muy pocos los casos en los que el planteo algebraico se conjuga con el gráfico (en el caso de las ALP). Con respecto a las $\mathrm{AE}$, predomina el uso de esquemas pero en pocos casos se propone la realización de gráficos cartesianos. Aun en los casos de experiencias de índole cualitativa, donde se estudia la relación entre dos variables que no se miden, se podría plantear a los alumnos que predigan cuál sería la forma del gráfico en el caso de que las mediciones se efectuaran.
BRINCONES, I. y OTERO, J. (1994). Students Conceptions of the Top-Level Structure of Physics Texts. Science Education, 78(2), pp. 171-183.

CABRAL DA COSTA, S. y MOREIRA, M.A. (1995). Resolução de problemas I: Diferenças entre novatos e especialistas. Memorias REF IX. Salta.

FEYNMAN, R. (1987). Física. Volumen II. EEUU: Addison Wesley.

GRÜNFELD, V. (1991). El caballo esférico. Buenos Aires: Editorial Lugar Científico. 
GUTIÉRREZ-VÁZQUEZ et al. (1993). Libros de texto y estilos de docencia. Uso de los libros de ciencias naturales en el estado de Michoacán. Revista Latinoamericana de estudios educativos, XXIII (4), pp. 87-107.

HODSON, D. (1985). Philosophy of Science, Science \& Science Education. Studies in Science Education, 12, pp. 25-57.

ISLAS, S. (1994). ¿Pueden cuestionarse las ideas previas de los estudiantes resolviendo problemas sobre un mundo ideal? Fifth Interamerican Conference on Physics Education. Texas. EEUU.

MATTHEWS, M.R. (1994). Historia, filosofía y enseñanza de las ciencias: aproximación actual. Enseñanza de las Ciencias, 12 (2), pp. 255-277.

MOREIRA, M. A. (1994). Cambio conceptual: crítica a modelos actuales y una propuesta a la luz de la teoría del aprendizaje significativo. Conferencia desarrollada en el II SIEF. Buenos Aires.

MICHINEL MACHADO, J. y D'ALESSANDRO MARTÍNEZ, A. (1994). El concepto de energía en los libros de texto: de las concepciones previas a la propuesta de un nuevo sublenguaje. Enseñanza de las Ciencias, 12(3), pp. 369-380.
OTERO, J. (1990). Variables cognitivas y metacognitivas en la comprensión de textos científicos: el papel de los esquemas y el control de la propia comprensión. Enseñanza de las Ciencias, 8(1), pp. 17-22

SALINAS DE SANDOVAL, J. y COLOMBO DE CUDMANI, L. (1993). Epistemología e historia de la física en la formación de los profesores de física. Revista Brasileira de Ensino de Física, 15 (1 a 4), pp.100-109.

SALINAS DE SANDOVAL, J., GIL PÉREZ, D. y COLOMBO DE CUDMANI, L. (1995). La elaboración de estrategias educativas acordes con un modo científico de tratar las cuestiones. Memorias REF IX. Salta.

SAMAJA, J. (1994). Epistemología y metodología. EUDEBA.

TAYLOR, S. y BOGDAN, R. (1992). Introducción a los métodos cualitativos de investigación. Barcelona: Paidós.

VICARIO, J. E. et al. (1995). ¿Contribuyen los libros de texto a la superación de los modelos representativos de los alumnos? Memorias REF IX. Salta.

VYGOTSKI, L. (1991). El desarrollo de los procesos psicológicos superiores. Barcelona: Crítica. Grijalbo.

[Artículo recibido en septiembre de 1996 y aceptado en noviembre de 1998.] 